\title{
IDENTIFICAÇÃO DE FRAGMENTOS DE FLORESTA NATIVA, POR DIFERENTES INTÉRPRETES, COM A UTILIZAÇÃO DE IMAGENS LANDSAT E CBERS EM LAVRAS, MG
}

\author{
Identification of small areas of semideciduous forest, by different analysts, \\ in Lavras region, MG, using Landsat and Cbers sattelites images
}

\author{
Elizabeth Ferreira $^{1}$, Jacinto Pereira Santos ${ }^{2}$, Antônio Carlos Barreto ${ }^{3}$, Antonio Augusto Aguilar Dantas ${ }^{4}$
}

\begin{abstract}
RESUMO
Neste trabalho, as imagens dos satélites Landsat 7 e Cbers 2 foram analisadas com o objetivo de identificar áreas com fragmentos de floresta semidecídua e de avaliar a exatidão da classificação feita por diferentes intérpretes e técnicas de interpretação. O estudo foi realizado em Lavras, MG, utilizando o SIG-SPRING, que possui recursos para realização da classificação digital e visual. Na comparação das diferentes classificações e avaliação da exatidão, foram empregadas as exatidões global, do consumidor, do produtor e o coeficiente Kappa. Pelos resultados, verificou-se que a exatidão global foi maior que $90 \%$ e o coeficiente Kappa variou de $50 \%$ a $77 \%$ nas comparações feitas por diferentes intérpretes, em imagens Landsat e Cbers. Os mapas de fragmentos de vegetação produzidos com base na classificação digital das imagens Cbers e Landsat apresentaram alta porcentagem de áreas comuns e os intérpretes produziram diferentes mapas, porém, aqueles gerados pela imagem Cbers apresentaram a melhor concordância entre as classificações.
\end{abstract}

Termos para indexação: sensoriamento remoto, índice Kappa, floresta semidecídua.

\begin{abstract}
In this work two images from Landsat 7 and Cbers 2 were analyzed in order to identify small areas of semideciduous forest and to evaluate the classification accuracy made by three different analysts. The study was carried out in Lavras region, MG, using the SPRING GIS with the appropriate functions to jufil the digital classification and visual inspection. The comparisons between the classifications and accuracy assessment procedures employed the overall accuracy, the user's accuracy, the producer's accuracy and the Kappa coefficient. The results showed that the overall accuracy were higher than $90 \%$ and the Kappa coefficient ranged from $50 \%$ to $77 \%$ when the Landsat and Cbers images were compared by different analysts. The fragments vegetation maps made from digital classification of Cbers and Landsat satellites images presented high percentage of common areas and analysts made different maps but, those one produced from Cbers satellite images were better than the other classifications.
\end{abstract}

Index terms: remote sensing, Kappa index, semideciduous forest.

(Recebido para publicação em 3 de Janeiro de 2005 e aprovado em 31 de março de 2005)

\section{INTRODUÇÃO}

A cobertura vegetal primitiva do sul do Estado de Minas Gerais, na região do Alto Rio Grande, foi reduzida a remanescentes e esparsas áreas, em sua maioria bastante perturbadas pelo fogo, pela pecuária extensiva ou pela retirada seletiva de madeira. As florestas semidecíduas, em particular, foram criticamente reduzidas, uma vez que sua ocorrência coincide com os solos mais férteis e úmidos, que são mais visados pela agropecuária (OLIVEIRAFILHO et al., 1994).

A ocupação das áreas desflorestadas foi estabelecida de forma desordenada, formando um complexo de pequenas e médias propriedades agrícolas com diversos usos, sendo os principais: pastagem, cultivo de café, cultivos anuais e reflorestamento. Esse padrão de uso e ocupação, aliado à topografia acidentada, dificulta a interpretação dos dados adquiridos por sensores remotos.

As imagens obtidas por sensores remotos registram a energia proveniente dos objetos da superfície observada na forma digital, a partir da qual um mapa poderá ser gerado com o uso de técnicas de interpretação visual ou automática (FLORENZANO, 2002).

\footnotetext{
1. Engenheira Agrícola, DSc., Setor de Cartografia - Professora do Departamento de Engenharia da Universidade Federal de Lavras/UFLA - Caixa Postal 3037 - 37200-000 - Lavras, MG.

2. Engenheiro Agrônomo, MSc., Fundação Universidade Tocantins/UFT.

3. Engenheiro Agrícola, MSc., CEFET, Uberaba.

4. Zootecnista, Dsc., Professor do Departamento de Engenharia/UFLA.
} 
Uma das principais vantagens da análise visual sobre a digital é a capacidade de incorporar critérios complexos à interpretação da imagem. A análise digital se baseia, quase exclusivamente, na intensidade radiométrica de cada pixel das bandas utilizadas na interpretação (CHUVIECO, 1990).

Dessa forma, as diferentes técnicas de interpretação e a escolha dos produtos (sensor, resolução, faixa espectral) influenciam as características dos mapas produzidos. Além desses fatores, os critérios de interpretação utilizados por diferentes intérpretes podem originar diferentes classificações. Focht (1998) utilizou o índice Kappa para avaliar a concordância de mapeamentos de capacidade de uso da terra produzidos por diferentes avaliadores.

Costa (1999) comparou diferentes classificadores automáticos, em uma imagem Landsat-TM, do município de Viçosa, MG, avaliando a porcentagem de área não classificada e o tempo de processamento, concluindo que o algorítmo de máxima verossimilhança foi o melhor, com exatidão global de $77 \%$.

Carvalho (2001) utilizou uma série de imagens Landsat-TM, da região do Alto do Rio Grande, MG, e diferentes técnicas de classificação e integração de dados, com o objetivo de discriminar remanescentes florestais de cultivos de café e eucalipto, obtendo exatidões que variaram de 34\% a 51\%.

Rudorff et al. (2004) fizeram estimativas de área plantada com cana-de-açúcar em municípios do Estado de São Paulo, mediante de imagens Landsat e modificaram o resultado da classificação digital, com interferência do intérprete para aumentar a exatidão do mapeamento temático final.

Segundo Congalton (1991), uma matriz de erro pode ser usada como ponto de partida para a aplicação de uma série de técnicas estatísticas descritivas e analíticas. Pela da estatística descritiva obtém-se o índice de exatidão global, em que se divide o total de pixels classificados corretamente (diagonal da matriz) pelo número total de pixels da matriz. A exatidão das categorias estudadas é feita de forma similar e o índice Kappa pode ser utilizado para inferir a exatidão desses mapeamentos.

Fidalgo (1995) utilizou vários métodos estatísticos propostos na literatura para estimar a exatidão de mapeamentos de remanescentes de Mata Atlântica, com a utilização de imagens Landsat-TM, produzidos por diferentes intérpretes e processos de digitalização, concluindo que a estimativa com o coeficiente Kappa para todos os produtos foram inferiores às respectivas estimativas de exatidão global.
Segundo Lands \& Koch (1977), a qualidade dos produtos, avaliada pelo índice Kappa, pode ser considerada boa (40-60\%), muito boa (60-80\%) e excelente (80-100\%).

As imagens de satélite de média resolução são comumente utilizadas no mapeamento de cobertura vegetal. As imagens dos satélites da série Landsat foram muito utilizadas, por apresentarem custo relativamente baixo e boa resolução temporal, gerando um consistente referencial para os usuários. Com a recente disponbilização das imagens do satélite sino- brasileiro Cbers, é fundamental que se construa um banco de dados referentes às informações obtidas por seus sensores, principalmente pela qualidade dessas imagens e porque o Landsat 7 encontra-se inoperante.

Neste trabalho, objetivou-se identificar, avaliar e comparar os mapeamentos de fragmentos de vegetação elaborados por diferentes intérpretes e técnicas de classificação com o uso de imagens dos satélites Landsat e Cbers.

\section{MATERIAL E MÉTODOS}

O trabalho foi realizado no município de Lavras, MG, com área total de 548,4024 km²,

localizado entre as coordenadas UTM $7.633 \mathrm{~km}$, $7.664 \mathrm{~km} \mathrm{~N}$ e $481 \mathrm{~km}, 514 \mathrm{~km}$ E, meridiano central 45 WGr.

Os mapas de fragmentos de floresta nativa foram elaborados por três intérpretes que utilizaram duas imagens de satélite e duas técnicas de interpretação, para as imagens Cbers.

As imagens dos satélites Landsat7-ETM+ e Cbers2-CCD, bandas 423/RGB de 26/06/00 e 04/07/04 foram selecionadas para o trabalho, por serem da mesma época do ano.

Os três intérpretes, utilizando critérios semelhantes para interpretação de imagens, elaboraram os mapas de fragmentos de vegetação utilizando independentemente a classificação digital supervisionada para imagens do Landsat-ETM+ e Cbers-CCD, e a interpretação visual para imagens do Cbers-CCD.

O trabalho foi realizado em três etapas. Na primeira etapa, os intérpretes reuniram-se para estabelecimento dos critérios a serem utilizados no desenvolvimento do trabalho e construíram um banco de dados básico no SPRING (CÂMARA et al., 1996).

Nesse banco, o projeto "Lavras" foi criado, contendo categorias no modelo imagem ("Imagem”), MNT (“Altimetria”) e temáticas ("Limite”, "Drenagem” e "Vegetação"). 
A base planialtimétrica (curvas de nível, estradas e cursos d'água), referentes às folhas Lavras, Itumirim, Carmo da Cachoeira e Nepomuceno, foram importadas dos arquivos digitais de cartas do IBGE. Efetuou-se o georreferenciamento da imagem Cbers pela captura de pontos de controle dessa base, como plano vetorial. A imagem Landsat foi registrada a partir da imagem Cbers georreferenciada, pelo processo imagem-imagem. Os planos de informação (PIs) criados dentro das categorias estão relacionados na Tabela 1.

Os intérpretes fizeram análises visuais de fragmentos de vegetação de mata nativa nas imagens, para estabelecerem uma chave de interpretação utilizando fotos aéreas e inspeções "in situ”. A principal fisionomia de vegetação identificada foi a florestal semidecídua que, segundo Oliveira-Filho \& Fluminham-Filho (1999), é encontrada no fundo dos vales, nas adjacências dos cursos d'água (mata ciliar) e encostas cujos solos são mais profundos (mata de encosta). Os fragmentos apresentaram-se em forma irregular, tamanho médio a pequeno, tonalidade vermelha escura, textura rugosa, localizados próximo às drenagens ou nas encostas.

Na classificação digital, utilizou-se o método supervisionado, adquirindo-se 20 amostras de treinamento, com limiar de aceitação de 99\%. O tamanho recomendado para a amostra de treinamento foi o menor polígono possível, com a imagem na escala 1:50.000. Não foi feita nenhuma transformação sobre as bandas espectrais e o critério utilizado para classificação foi o de máxima verossimilhança.

$\mathrm{Na}$ interpretação visual, utilizou-se o módulo escala 1:50.000, para a digitalização das feições na tela do computador.

Após a realização da segunda etapa, que consistiu da elaboração dos mapeamentos de fragmentos de vegetação pelos intérpretes, os arquivos (PIs) foram todos importados para o banco de dados básico, como pode ser visto na categoria vegetação da Tabela 1 .

Na terceira etapa do trabalho, as áreas menores que $5000 \mathrm{~m}^{2}$ foram eliminadas em todos os PIs, sendo esses transformados para o formato matricial com resolução espacial de 30 x 30 m, resultando em imagens de 1847 x 1738 pixels.

As análises espaciais de concordância e conflito entre as áreas mapeadas foram feitas utilizando-se da função tabulação cruzada e do programa Legal.

A comparação entre os produtos foi feita com uso dos índices de exatidão global e Kappa (CHUVIECO, 1990; CONGALTON, 1991; FIDALGO, 1995). Os produtos foram finalizados utilizando os módulos Scarta e Iplot do SPRING.

\section{RESULTADOS E DISCUSSÃO}

Os mapas de fragmentos de vegetação elaborados com base em diferentes imagens de satélite, intérpretes e métodos apresentaram áreas totais e número de polígonos diferentes, conforme pode ser observado na Tabela 2.

A classificação digital (Tabela 2) gerou 10\% e 8\%, em média, de áreas com fragmentos de vegetação, respectivamente, para as imagens Landsat e Cbers. Esse fato pode ter ocorrido devido a um problema apresentado na parte noroeste da imagem Cbers (Figura 1), uma vez que os mapas de todos os intérpretes não mostraram essa área classificada, para as imagens Cbers. A interpretação visual permitiu mostrar que o menor número de polígonos e área total dos fragmentos de vegetação, obtida pelos diferentes intérpretes, foi inferior à obtida pela classificação digital, em média 6\%.

TABELA 1 - Planos de Informação do Projeto “Lavras”, elaborados nas Etapas 1 e $2^{(*)}$

\begin{tabular}{llc}
\hline \multicolumn{1}{c}{ Modelo } & Categoria & Planos de Informação (PIs) \\
\hline Imagem & Imagem & Cbers_B2, Cbers_B3, Cbers_B4 \\
& & Landsat_B2, Landsat_B3, Landsat_B4 \\
MNT & Altimetria & Curvas_de_nível \\
Temático & Limite & Divisa_município \\
Temático & Drenagem & Cursos_d'água \\
\hline Temático $^{(*)}$ & Vegetação & Land_int1_digital; Cbers_int1_digital; \\
& & Land_int2_digital; Cbers_int2_digital; \\
& & Land_int3_digital; Cbers_int3_digital; \\
& & Cbers_int1_visual; Cbers_int2_visual; Cbers_int3_visual; \\
\hline
\end{tabular}


TABELA 2 - Áreas ( $\mathrm{km}^{2}$ e porcentagem) e número de polígonos produzidos pelas diferentes imagens de satélite, intérpretes e métodos de classificação.

\begin{tabular}{lccccccccc}
\hline Satélite & Landsat & Landsat & Landsat & Cbers & Cbers & Cbers & Cbers & Cbers & Cbers \\
\hline Intérprete & 1 & 2 & 3 & 1 & 2 & 3 & 1 & 2 & 3 \\
Classificação & Digital & Digital & Digital & Digital & Digital & Digital & Visual & Visual & Visual \\
Área $\left(\mathrm{km}^{2}\right)$ & 70,32 & 35,64 & 58,30 & 50,41 & 54,15 & 31,91 & 24,5 & 35,63 & 34,73 \\
Área (\%) & 13 & 7 & 11 & 9 & 10 & 6 & 4 & 7 & 7 \\
Polígonos & 1759 & 1255 & 1647 & 1493 & 1634 & 1084 & 388 & 368 & 366 \\
\hline
\end{tabular}

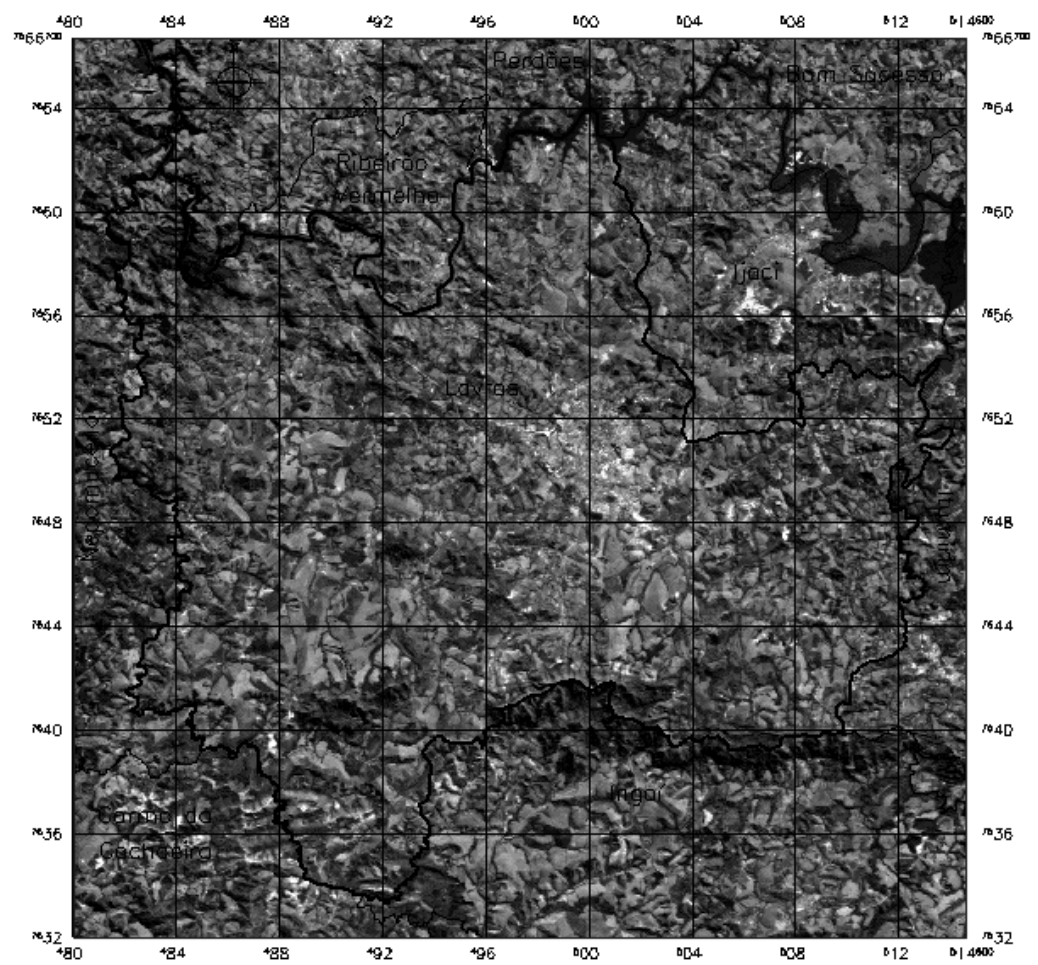

FIGURA 1 - Imagem do satélite Cbers-CCD, composição colorida 423/RGB, órbita/ponto 152/124, município de Lavras, MG, em 04/07/2004.

Na Tabela 3 verifica-se uma matriz de erro, com avaliação dos índices de exatidão global, exatidão do consumidor, exatidão do produtor e índice Kappa, quando diferentes imagens de satélite foram utilizadas pelo mesmo intérprete, por meio de classificação digital. Nesse caso, as imagens Lansat foram utilizadas como referência para a análise das imagens Cbers.

Os índices dessa tabela indicam a probabilidade de uma área de vegetação classificada na imagem
Landsat ter sido também classificada na imagem Cbers, quando analisada por três intérpretes. Os índices de exatidão global foram de 96, 93 e 92\%, respectivamente, para os intérpretes 1, 2 e 3 . Esses resultados permitiram concluir que esse índice foi excelente e que as imagens Landsat e Cbers são adequadas para a classificação digital, mesmo quando manipuladas por diferentes usuários ou intérpretes. No entanto, Congalton (1991) alerta que, com base apenas 
no índice de exatidão global, as inferências feitas podem gerar equívocos e conclui que o cálculo e a análise da "user's accuracy" confere melhor significado aos dados, revelando se há confusão entre as categorias. Fidalgo (1995) denomina de "exatidão do consumidor" o termo "user's accuracy”, e “exatidão do produtor” o termo “producer's accuracy”, utilizados por Congalton (1991).

Os dados mostram valores de exatidão do produtor de 95, 45 e 76\%, respectivamente, para os intérpretes 1, 2 e 3 (Tabela 3) e de exatidão do consumidor de 68, 69 e 41\%.

Esses dados indicam que para o intérprete 1, apesar de 95\% dos dados da imagem Cbers terem sido classificadas como floresta, isso correspondeu a 68\% da vegetação classificada na imagem Landsat; para o intérprete 2, apenas $45 \%$ dos dados da imagem Cbers foi classificada como floresta e isto correspondeu a 69\% de floresta na imagem Landsat; para o intérprete 3, 76\% dos dados da imagem Cbers foram classificados como floresta, sendo esses correspondentes a $41 \%$ da floresta classificada na imagem Landsat.

O índice Kappa, utilizado para avaliar a exatidão ou concordância, resultou em valores de 77, 51 e 50\%, respectivamente, para os intérpretes 1, 2 e 3 . Os resultados desse índice revelam que houve diferenças entre os mapas produzidos pelos diferentes intérpretes, quando foi utilizada imagens Cbers e Landsat, sendo a melhor concordância conseguida pelo intérprete 1. A qualidade dos mapas produzidos e comparados obtiveram uma avaliação boa pelo índice Kappa para os intérpretes 2 e 3, e muito boa, para o intérprete 1 (LANDS \& KOCH, 1977). Na Figura 2 pode ser observada a distribuição espacial das áreas de fragmentos florestais coincidentes e não coincidentes (conflito), obtidas pelo intérprete 1.

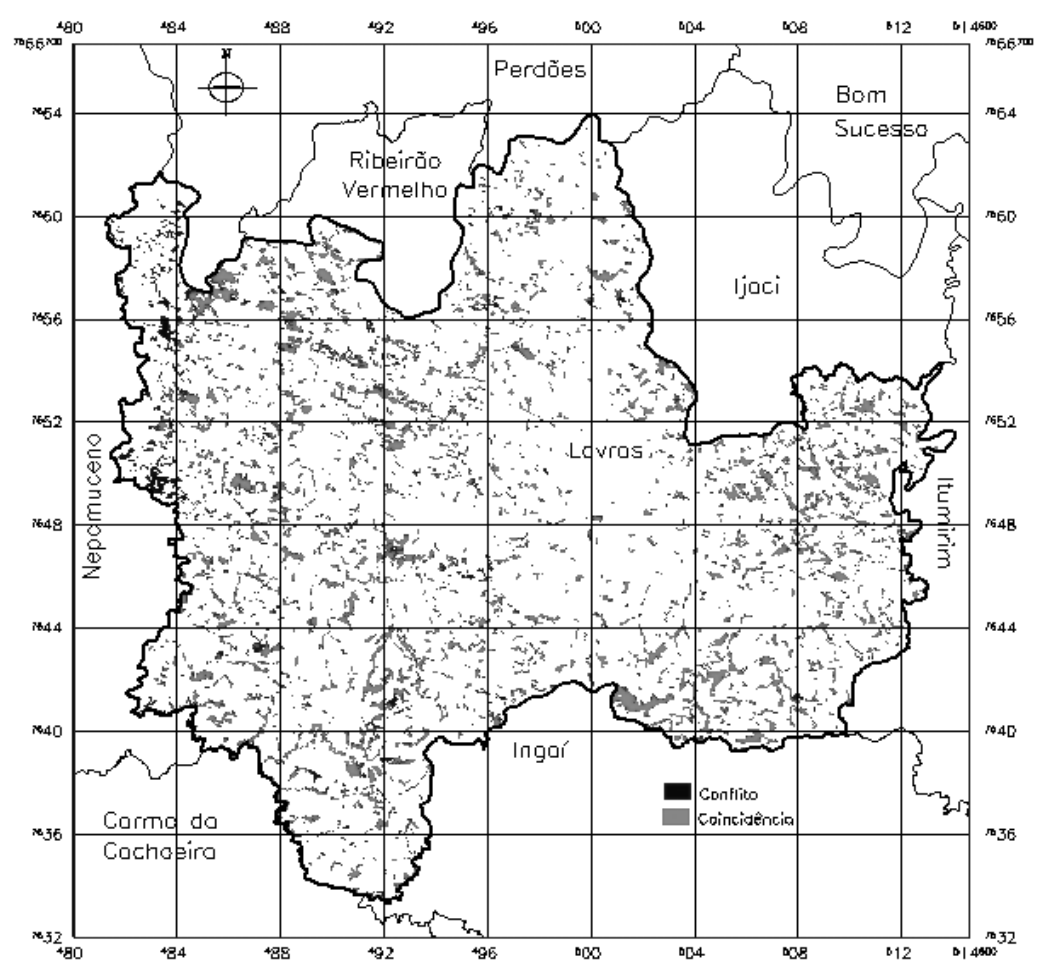

FIGURA 2 - Áreas de fragmentos florestais coincidentes e não coincidentes (conflito) obtidas a partir das imagens Cbers e Landsat, com índice Kappa $=77 \%$, município de Lavras, MG. 
TABELA 3 - Índice de exatidão global, exatidão do consumidor, exatidão do produtor e índice Kappa, em porcentagem, para imagens Landsat (na linha) e Cbers (coluna).

\begin{tabular}{|c|c|c|c|c|}
\hline Cbers & & Landsat & & \\
\hline Intérprete 1 & Mata & Outros & Total & Ex. do Consumidor \\
\hline Mata & 48,1086 & 22,2174 & 70,326 & $68 \%$ \\
\hline Outros & 2,3004 & 475,576 & 477,8764 & $100 \%$ \\
\hline Total & 50,409 & 497,7934 & 548,2024 & \\
\hline Ex. do produtor & $95 \%$ & $96 \%$ & & \\
\hline Exatidão Global & $=96 \%$ & & & Карра = 77\% \\
\hline Cbers & & Landsat & & \\
\hline Intérprete 2 & Mata & Outros & Total & Ex. do Consumidor \\
\hline Mata & 24,5655 & 11,079 & 35,6445 & $69 \%$ \\
\hline Outros & 29,5884 & 482,9695 & 512,5579 & $94 \%$ \\
\hline Total & 54,1539 & 494,0485 & 548,2024 & \\
\hline Ex. do produtor & $45 \%$ & $98 \%$ & & \\
\hline Exatidão Global = & $93 \%$ & & & Kappa $=51 \%$ \\
\hline Cbers & & Landsat & & \\
\hline Intérprete 3 & Mata & Outros & Total & Ex. do Consumidor \\
\hline Mata & 24,1074 & 34,2 & 58,3074 & $41 \%$ \\
\hline Outros & 7,7994 & 482,0956 & 489,895 & $98 \%$ \\
\hline Total & 31,9068 & 516,2956 & 548,2024 & \\
\hline Ex. do produtor & $76 \%$ & $93 \%$ & & \\
\hline Exatidão Global = & $92 \%$ & & & Карра $=50 \%$ \\
\hline
\end{tabular}

As matrizes de erro foram construídas (Tabela 3) para avaliar a exatidão produzida pelos diferentes intérpretes, quando utilizados diferentes produtos. Nesse caso, foram utilizados os índices de exatidão global e Kappa, já que um intérprete não pode ser considerado referência em relação ao outro (Tabela 4).
Na Tabela 4 pode ser observado que, independente da imagem utilizada, os índices de exatidão global foram maiores que os índices Kappa. Resultados semelhantes foram encontrados por vários autores (COSTA, 1999; CONGALTON, 1991; FIDALGO, 1995). 
A análise dos índices Kappa (Tabela 4) e a concordância entre os intérpretes variaram de boa (49\% a $60 \%$ ), quando foram utilizadas as técnicas de classificação digital, para imagens Landsat, e visual, para imagens Cbers. A concordância foi considerada muito boa (72\% e 67\%) e excelente (88\%) quando foi utilizada a classificação digital em imagens do Cbers. O resultado das operações de intersecção (coincidências) e disjunção (conflito) realizada entre os PIs Cbers_int1_digital; Cbers_int2_digital e Cbers_int3_digital (Figura 3). As áreas de coincidência corresponderam a $29,3274 \mathrm{~km}^{2}$, ou seja, 5\% da área do município. A interação entre todos produtos gerou $8,1054 \mathrm{~km}^{2}$ de coincidência e 58,886 $\mathrm{km}^{2}$ de conflito.

A comparação entre os mapas produzidos pelo mesmo intérprete, utilizando as classificações digital e visual, para as imagens Cbers, resultou em índices Kappa de 53\%, 51\% e $46 \%$, respectivamente, para os intérpretes 1 , 2 e 3 . Para os respectivos intérpretes, os índices de exatidão do produtor foram de $41 \%$, $45 \%$ e $52 \%$ e do consumidor de $85 \%$, $69 \%$ e $48 \%$. Nesse caso, o intérprete 3 foi o que apresentou maior discordância entre as classificações.

TABELA 4 - Índices de exatidão global e Kappa, em porcentagem, produzidos por diferentes imagens de satélite e intérpretes.

\begin{tabular}{cccccccccc}
\hline Satélite & Landsat & Landsat & Landsat & Cbers & Cbers & Cbers & Cbers & Cbers & Cbers \\
\hline Intérpretes & 1 e 2 & 1 e 3 & 2 e 3 & 1 e 2 & 1 e 3 & 2 e 3 & 1 e 2 & 1 e 3 & 2 e 3 \\
Classificação & Digital & Digital & Digital & Digital & Digital & Digital & Visual & Visual & Visual \\
Ex. Global & 91 & 91 & 94 & 98 & 96 & 95 & 95 & 95 & 94 \\
Kappa (\%) & 49 & 56 & 60 & 88 & 72 & 67 & 54 & 54 & 49 \\
\hline
\end{tabular}

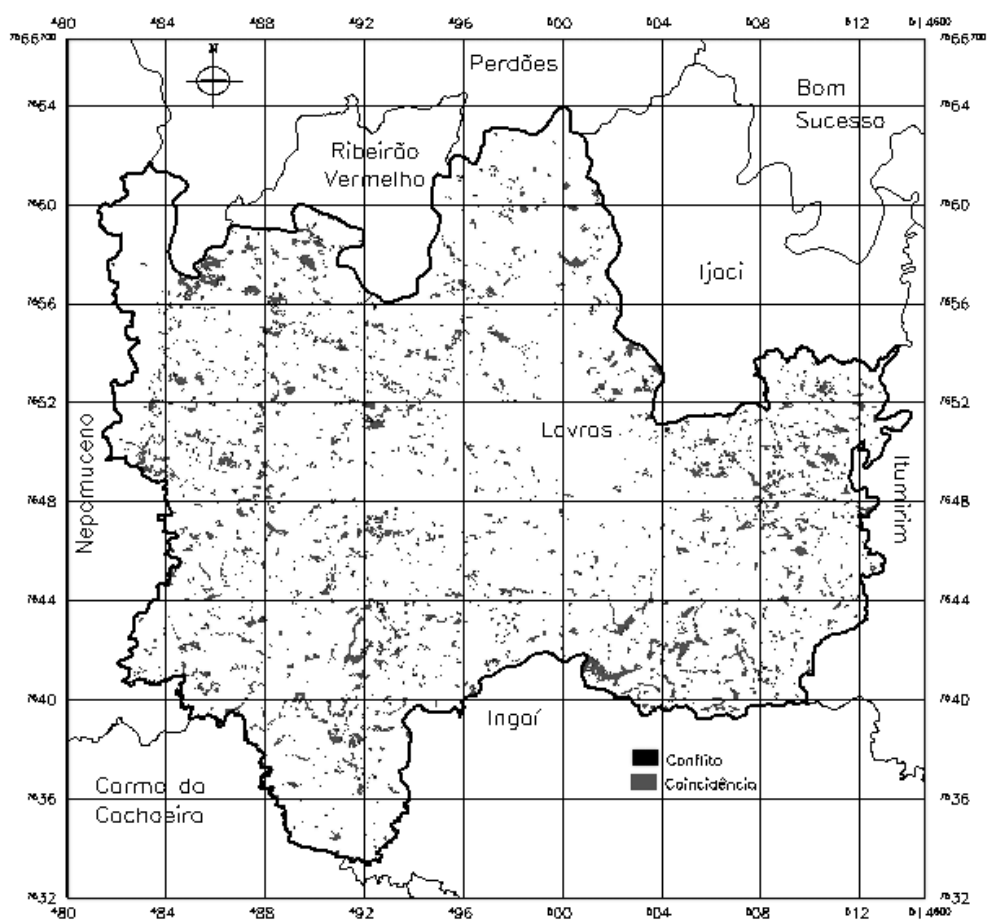

FIGURA 3 - Áreas de fragmentos florestais coincidentes e não coincidentes (conflito), obtidas a partir da classificação digital feita por três intérpretes, através da imagem Cbers, município de Lavras, MG. 


\section{CONCLUSÕES}

Com base nos resultados obtidos, pode-se concluir que os mapas de fragmentos de vegetação produzidos a partir da classificação digital das imagens Cbers e Landsat apresentaram uma boa concordância e os intérpretes produziram diferentes mapas; porém, aqueles gerados a partir da imagem Cbers apresentaram a melhor concordância entre as classificações.

A interpretação visual da imagem Cbers apresentou menos áreas de fragmentos de vegetação do que as obtidas pela classificação digital.

Os produtos Cbers podem ser utilizados com segurança para substituir as imagens Landsat7, que foram descontinuadas.

\section{REFERÊNCIAS BIBLIOGRÁFICAS}

CÂMARA, G.; SOUZA, R. C. M.; FREITAS, U. M.; GARRIDO, J. Spring: integrating remote sensing and GIS by object-oriented data modelling. Computers \& Graphics, [S.l.], v. 20, n. 3, p. 395-403, 1996.

CARVALHO, L. Mapping and monitoring forest remnts: a multi-scale analysis of spatio-temporal data. 2001. 140 f. Thesis (Doctor) - Wagenigen Universiteit, Wagenigen , 2001.

CONGALTON, R. G. A review of assessing the accuracy classificatons of remotely sensed data. Remote Sensing Environment, [S.I.], v. 37, p. 35-46, 1991.

COSTA, T. C. e C. da. Avaliação de estratégias para classificação de imagens orbitais em mapeamento de uso e cobertura da terra. 1999. 109 f. Tese (Doutorado em Ciência Florestal) - Universidade Federal de Viçosa, Viçosa, 1999.

CHUVIECO, E. Fundamentos de teledection espacial. Madrid: Unigraf, 1990. 453 p.
FIDALGO, E. C. C. Exatidão no processo de mapeamento temático da vegetação de uma área de mata atlântica no estado de São Paulo, a partir de imagens TM-Landsat. 1995. 186 f. Dissertação (Mestrado em Sensoriamento Remoto) - Instituto Nacional de Pesquisas Espaciais, São José dos Campos, 1995.

FOCHT, D. Influência do avaliador no resultado da classificação de terras em capacidade de uso. 1998. 79 f. Dissertação (Mestrado em Agronomia) - Escola Superior de Agricultura "Luiz de Queiroz", Piracicaba, 1998.

FLORENZANO, T. G. Imagens de satélite para estudos ambientais. São Paulo: Oficina de textos, 2002. $97 \mathrm{p}$.

LANDS, J. R.; KOCH, G. G. The measurement of observer agreement for categorial data. Biometrics, Washington, v. 33, p. 159-154, 1977.

OLIVEIRA-FILHO, A. T.; FLUMINHAN-FILHO, M. Ecologia da vegetação do parque florestal do Rio Bonito. Cerne, Lavras, v. 5, n. 2, p. 51-64, 1999.

OLIVEIRA-FILHO, A. T.; VILELA, E. A.; GAVILANES, M. L.; CARVALHO, D. A. Comparison of the woody flora and soils of six areas of montane semideciduous forest in southern Minas Gerais, Brazil. Edinburgh Journal of Botany, Edinburgh, v. 51, n. 3, p. 355-389, 1994.

RUDORFF, B. F. T.; BERKA, L. M. S.; MOREIRA, M. A.; DUARTE, V.; ROSA, V. G. C. Estimativa de área plantada com cana-de-açúcar em municípios do estado de São Paulo por meio de imagens de satélite e técnicas de geoprocessamento: ano 2004-2005. São Paulo: INPE, 2004. 